\title{
FULLANALYZE: A RESEARCH TOOL FOR HANDLING, PROCESSING AND ANALYZING FULL-WAVEFORM LIDAR DATA
}

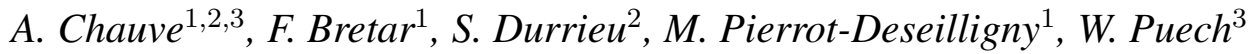 \\ ${ }^{1}$ Laboratoire MATIS - Institut Géographique National, 2-4 Avenue Pasteur, 94165 Saint Mandé cedex, France \\ ${ }^{2}$ UMR TETIS Cemagref/Cirad/ENGREF-AgroParisTech, Maison de la Télédétection, 34093 Montpellier Cedex 5, France \\ ${ }^{3}$ Laboratoire LIRMM, UMR CNRS 5506, Université Montpellier II, 161, rue Ada, 34392 Montpellier Cedex 05, France
}

\section{INTRODUCTION AND BACKGROUND ON FULL-WAVEFORM LIDAR DATA}

Airborne lidar systems (ALS) provide 3D point clouds of the topography by direct time measurement of a short laser pulse after reflection on the Earth surface. For the last decade, this technique has proved to be the ideal remote sensing tool for delivering very accurate digital terrain model (DTM) of the Earth surface, and then for answering main environmental issues such as natural hazard prevention and natural ressource management. Moreover, such active systems, also called "multiple echo lidar", allow to detect several return signals for a single laser shot. It is particularly relevant in case of vegetation areas since a single lidar survey allows to acquire not only the canopy top (the only visible layer from passive sensors), but also points inside the vegetation layer and on the ground underneath. Thus, among the different remote sensing techniques, airborne laser scanning has also proved to be the most efficient technique to characterize both forest structure and ground topography.

For a few years, new airborne laser scanning systems called full-waveform lidar systems have emerged, providing not only 3D point clouds as classical ALS systems, but entire altimeter profiles of reflected energy from the Earth surface. These profiles represent the laser backscattered energy as a function of time. They give to the end-user more control and flexibility on the signal processing steps and enable to extract more information than classical multi-echo lidar data. A detailed state-of-the-art of such systems can be found in [1]. However, managing these data with spacial and time dependency is much more complex than images or 3D point clouds : raw full-waveform lidar data are sets of range profiles of various lengths that are stored in the sensor geometry following both the scan angle of the lidar system and the chronological order along the flight track. Moreover, the data volume is drastically larger than 3D point clouds: it takes about $140 \mathrm{~GB}$ for an acquisition time of $1.6 \mathrm{~h}$ with a pulse repetition frequency (PRF) of $50 \mathrm{kHz}$. Furthermore, there is neither commercial nor opensource toolkit to handle full-waveform lidar data, but some constructor solutions, that are black boxes, can only extract 3D point clouds from raw data and are designed to their own sensors. Finally, there is not standard file format for full-waveform data (such as the LAS format for multi-echo data). Managing full-waveform lidar data is therefore a challenging task, and we adress this issue by developping a specific research tool: FullAnalyze.

\section{MANAGING FULL-WAVEFORM LIDAR DATA}

The research tool FullAnalyze is written in $\mathrm{C}++$ and has been developed to provide the following functionalities.

\subsection{Handling raw data and georeferencing}

Raw full-waveform lidar data are stored in proprietary file formats which are sensor dependent. The first processing is then to georeference these altimeter profiles and calculate a direction and an origin for each profile first in a given geodetic datum, then in a cartographic projection. These georeferenced full-waveform data are then converted into a generic and open full-waveform file format. Currently data from the RIEGL(C) sensor LMSQ-560 have been successfully handled, and data from an OPTECH(C) sensor are in progress. Large footprint full-waveform lidar data from satelite profilers should also be integrated.

\subsection{Visualization}

Full-waveform lidar data can be visualized in FullAnalyze as 1D signal in the sensor topology (scan angle and time), but they can also be visualized in a 3D cartographic view superimposed on classical 3D point clouds to help interpreting and 
understanding the data. Indeed, the complex interaction between the laser waveforms and the topography (optical properties of the encountered materials, terrain slope etc.) can firstly be infered by a close and detailed visual analysis of the spacial and time variability of the acquired data.

\subsection{Waveform processing}

The first method used for waveform processing in FullAnalyze is based on a paper written by [2]. The technique relies on a parametric approach to fit the waveform and extract relevant peaks as echoes. This allows to extract $\mathrm{x}, \mathrm{y}, \mathrm{z}$ position but also additional information about the target shape and its reflectance. This method has proved very efficient in forested areas enabling to detect a lot of additional echoes compared to lidar operator treatments [3], but it is also useful in urban areas where additional parameters of the modeled waveforms lead to improved classification results [4].

Another method implemented in FullAnalyze is to keep the entire waveforms and sum them in a given neighborhood larger than just one small footprint. The aim is to analyze full-waveform information at a higher scale than a footprint (typically for objects such as trees). A radiative transfer model can then be inverted to compute a height profile of projected plant area, as done for large footprint data [5].

\subsection{Algorithms}

The aim of FullAnalyze is also to be modular using a plugin architecture so as to be able to easily add additional algorithms working on full-waveform lidar data. This architecture has been tested with a 3D landcover classification algorithm based on SVM (Support Vector Machine). The inputs are 3D point clouds with additional features extracted from the waveform processing step and ortho-images if available.

\section{CONCLUSIONS}

FullAnalyze has been successfully used to handle and process small footprint full-waveform lidar data. This research tool should be fully available in open-source before the end of 2009.

\section{REFERENCES}

[1] C. Mallet and F. Bretar, "Full waveform topographic lidar: State-of-the-art," ISPRS Journal of Photogrammetry and Remote Sensing, in press.

[2] A. Chauve, C. Mallet, F. Bretar, S. Durrieu, M. Pierrot-Deseilligny, and W. Puech, "Processing full-waveform lidar data: modelling raw signals," in International Archives of Photogrammetry, Remote Sensing and Spatial Information Sciences, Espoo, Finland, Sept. 2007, vol. 36 (Part 3/W52), pp. 102-107.

[3] A. Chauve, C. Vega, F. Bretar, S. Durrieu, T. Allouis, M. Pierrot-Deseilligny, and W. Puech, "Processing full-waveform lidar data in an alpine coniferous forest: Assessing terrain and tree height quality," International Journal of Remote Sensing, accepted.

[4] C. Mallet, U. Sörgel, and F. Bretar, "Analysis of full-waveform lidar data for classification of urban areas," Photogrammetrie Fernerkundung GeoInformation (PFG), vol. 5, pp. 337-349, 2008.

[5] D.J. Harding, M.A. Lefsky, and G.G. Parker, "Laser altimeter canopy heigh profiles. Methods and validation for closedcanopy, broadleaf forests," Remote Sensing of Environment, vol. 76, no. 9, pp. 283-297, 2001. 